\title{
ESTUDO EXPLORATÓRIO SOBRE OS MÉTODOS OPM3 E KPMMM PARA AVALIAÇÃO DE MATURIDADE ORGANIZACIONAL NO GERENCIAMENTO DE PROJETO
}

\section{EXPLORATOTY STUDY ABOUT THE METHODS OPM3 AND KPMMM FOR EVALUATION ORGANIZATIONAL MATURITY IN PROJET MANAGEMENT}

\author{
Evandro Luiz de Oliveira ${ }^{1}$; Edson Aparecida de Araújo Querido de Oliveira ${ }^{2}$ \\ ${ }^{1}$ Universidade de Taubaté (UNITAU) - Taubaté - SP \\ evandro.l.o@ig.com.br \\ ${ }^{2}$ Universidade de Taubaté (UNITAU) - Taubaté - SP \\ edsonquerido@terra.com.br
}

\begin{abstract}
Resumo
O gerenciamento de projetos tem sido uma abordagem com acelerada difusão nas últimas três decadas nas organizações de diversos segmentos do mercado no Brasil e no mundo. Apesar da ampla divulgação e utilização, ainda é alto o índice de insucesso ou sucesso parcial na execução dos emprendimentos. Como esse campo de estudo é recente, levou-se ao questionamento se esse indice é consequência da imaturidade das organizações na utilização dos conhecimentos, técnicas $e$ habilidades dessa área. Este estudo, realizado a partir de pesquisa bibliográfica, tem como objetivo comparar e identificar as diferenças conceituais dos modelos Organizational Project Management Maturity Model ${ }^{\circledR}\left(\mathrm{OPM}^{\circledR}\right)$ e Kerzner Project Managemente Maturity Model ${ }^{\circledR}$ $\left(K_{\left.P M M M^{\circledR}\right)}\right.$ utilizadas para avaliação da maturidade em gerenciamento de projetos nas organizações. A comparação dos métodos $O P M 3^{\circledR}$ e KPMMM ${ }^{\circledR}$, com base em revisão bibliográfica, permite concluir que há complementaridade entre as duas abordagens e algumas diferenças, o que possibilita que o modelo KPMMM ${ }^{\circledR}$ possa ser aplicado em combinação com o OPM $3^{\circledR}$, para assim maximizar os resultados ao mesmo tempo definir um foco para as primeira análises, o que possibilita economizar, recursos e reduzir o tempo para obter indicações para melhorias.
\end{abstract}

Palavras chaves: maturidade em gestão de projetos; método OPM ${ }^{\circledR}$; método $\mathrm{KPMMM}^{\circledR}$.

\section{Introdução}

O mundo contemporâneo, para dar conta dos desafios e aproveitar o desenvolvimento das ciências, conhecimento e expansão das riquezas, vem realizando empreendimentos em para possibilitar melhor aproveitamento e distribuição dos bens produzidos. As dimensões econômicas, técnicas e sociais desses empreendimentos desafiam os conhecimentos e habilidades dos líderes, gestores, acadêmicos, pesquisadores, consultores, cientistas e trabalhadores em todo o mundo, o que 
levou ao desenvolvimento de uma nova disciplina ou área de conhecimento, denominada Gerenciamento de Projetos. Essa disciplina, com as contribuições realizadas pela comunidade acadêmica, pesquisadores e profissionais de diversos segmentos, desde seu surgimento na década de 1960, permitiu o desenvolvimento das várias abordagens disponíveis atualmente, além da ampliação e difusão da utilização nas organizações contemporâneas (MAXIMIAMO, 2010; MERDITH e MANTEL, 2009; CARVALHO e RABECHINNI, 2008; WEBSTER e KNUSTON, 2006).

Como resultado da associação dos profissionais atuantes na gestão execução de empreendimentos de diversos setores, com os objetivos de difundir, consolidar e ampliar os conhecimentos e técnicas para realizar do gerenciamento de projetos com volume e dimensões em crescimento no mercado mundial, em 1969 foi fundado nos Estados Unidos da América, com sede no estado da Filadélfia, o Project Management Institute $\left(\mathrm{PMI}^{\circledR}\right)$. Essa organização publica e tem reservado os direitos autorais da obra 'A Guide to the Project Management Body of Knowledge' ou, simplesmente, como é mais conhecida, PMBOK $^{\circledR}$ Guide, ou Guia PMBOK ${ }^{\circledR}$ (PMI, 2008a; DINSMORE e CAVALIERI, 2009; VALLE et al 2010; KERZNER, 2009). Essa obra é sintetize das técnicas, ferramentas e práticas amplamente aceitas para constituir-se uma abordagem que se encontra entre as mais utilizadas mundialmente na gestão de projetos.

Os profissionais, pesquisadores, e as organizações envolvidas com a execução de projetos, ao mesmo tempo em que reconhecem a importância e a relevância da gestão de projetos para os negócios, passaram a questionar como atingir e qual o significado de uma organização madura em gerenciamento de projetos.

Em um esforço para atender essa necessidade e trazer ao mundo dos negócios referenciais para avaliar e definir planos de ação voltados ao incremento da maturidade em gerenciamento de projetos nas companhias, o Project Manager Institute ${ }^{\circledR}\left(\mathrm{PMI}^{\circledR}\right)$ lançou em 2003 a primeira versão da norma OPM $3^{\circledR}$ - Organization Project Management Maturity Model, e publicou em 2008 a segunda edição (PMI $\left.{ }^{\circledR}, 2008\right)$.

Antes do lançamento da norma OPM3 ${ }^{\circledR}$ pelo PMI ${ }^{\circledR}$ (2008), o Dr. Harold Kerzner publicou, em 2001, um modelo de avaliação de maturidade em gerenciamento de projetos denominado Kerzner Project Management Maturity Model (KPMMM ${ }^{\circledR}$ ), tendo sido revisado e publicada uma segunda edição em 2005, com o lançamento do livro Using the Project Manager Maturity Model: Strategic Planning for Project Management, publicado pela editora John Wiley \& Sons e com suporte do International Institute for Learning, conhecido pela sigla ILL (KERZNER, 2005).

A análise é corroborada por Carvalho e Rabechini (2010), ao constatarem que as abordagens de gerenciamento de projetos têm apresentado crescimento e se difundido por diversos segmentos ou setores da economia, além da importância e empenho dos profissionais, comunidade acadêmica 
e entidades envolvidas em amadurecer e ampliar a utilização eficaz das técnicas e ferramentas para realizarem esse gerenciamento.

A partir da análise desse panorama identificou-se a relevância de desenvolver uma pesquisa bibliográfica referente a esses dois métodos.

\section{Referencial teórico}

\subsection{Maturidade em gerenciamento de projetos}

No dicionário Aurélio (2004) encontra-se a definição de maturidade como algo "plenamente desenvolvido; completamente formado". Essa definição é corroborada pela do dicionário Houassis (2009), que define a maturidade como a "que se acha em estado de produzir o resultado esperado".

Conforme observado por Andersen e Jessen (2003, p. 457), pode-se concluir que "maturidade é a qualidade ou estado de ser maduro". Quando se aplica esse conceito a uma organização, indica que é plenamente desenvolvida e, portanto, apta a atingir seus objetivos.

A maturidade em gerenciamento de projetos, por sua vez, expressa o entendimento que a organização é capaz de executar seus projetos e atingir seus objetivos organizacionais (ANDERSEN e JESSEN, 2003; PRADO, 2010, ARCHIBALD, 2005).

Kerzner (2009, p. 58) define maturidade em gerenciamento de projetos como "a implementação de um método padrão e um processo de acompanhamento de modo que exista uma alta probabilidade de repetidos sucessos". Essa definição é coerente com as anteriores ao atribuir os resultados à maturidade.

Segundo Kerzner (2002), em gestão de projetos existem dois tipos básicos de organização: as maduras e as imaturas, cujas características são apresentadas no Quadro 1.

Quadro 1 - Características das empresas maduras e imaturas

\begin{tabular}{|l|l|}
\hline \multicolumn{1}{|c|}{ Madura } & \multicolumn{1}{c|}{ Imatura } \\
\hline $\begin{array}{l}\text { A gestão de projetos é vista como um instrumento } \\
\text { para o sucesso, não como uma interferência nos } \\
\text { objetivos do dia a dia. }\end{array}$ & $\begin{array}{l}\text { A gestão de projetos é vista como um processo de } \\
\text { intimidação e de perturbação das operações do dia a } \\
\text { dia. }\end{array}$ \\
\hline $\begin{array}{l}\text { As mudanças organizacionais na estrutura } \\
\text { promovidas pela gestão de projetos conduziram à } \\
\text { maximização da utilização dos recursos humanos. }\end{array}$ & $\begin{array}{l}\text { Surgiram disputas pelo poder relacionadas com a } \\
\text { alocação dos escassos recursos humanos e técnicos. }\end{array}$ \\
\hline $\begin{array}{l}\text { A sinergia está presente na gestão de projetos. } \\
\text { Toda a empresa apoia o/a gerente de projetos e } \\
\text { suas exigências. }\end{array}$ & $\begin{array}{l}\text { Os executivos e gerentes de projetos tendiam a } \\
\text { administrar paroquialmente se o pessoal das áreas e } \\
\text { suas especialidades. }\end{array}$ \\
\hline $\begin{array}{l}\text { são havia uma base de planejamento para evolução } \\
\text { formal versus informal das estruturas de gestão de } \\
\text { projetos. Não havia estudo algum sobre qual seria a } \\
\text { melhor estrutura para um projeto antes de seu início. }\end{array}$ \\
\hline
\end{tabular}

Fonte: adaptado de Kerzner (2002) 
Segundo Kerzner (2002), a transformação de uma organização imatura para madura em gerenciamento de projetos depende do apoio da alta administração e de cada uma percorrer seu caminho para a maturidade, um processo que é medido em anos.

Essa percepção de maturidade como processo é compartilhada por Andersen e Jessen (2003) ao observarem que implícito ao conceito de maturidade está à possibilidade de usá-la como uma avaliação da capacidade de a organização usar projetos para diversos objetivos, e que a maturidade é desenvolvida de um patamar para outro mais elevado.

O conceito de maturidade é complementado pelo conceito de excelência, considerando a advertência de Kerzner (2002), segundo o qual a existência de processos e sistemas padronizados para o gerenciamento de projetos aumenta a probabilidade de sucesso, mas não o garante.

As organizações excelentes em gerenciamento de projetos são definidas por Kerzner (2009) como, "aquelas que criaram um ambiente no qual existe um continuo fluxo de projetos gerenciados e onde o sucesso é medido pelo o que é o melhor interesse de ambos da companhia e do projeto (isto é o cliente)" (KERZNER, 2009, p. 58).

Ao se analisar a representação esquemática na Figura 1, dos conceitos de maturidade e excelência segundo Kerzner (2009), compreende-se a observação de Kerzner (2009) de que a maturidade precede a excelência e que a excelência vai além de maturidade. Por outro lado, o autor também adverte que, "se não documentar as lições apreendidas, a empresa pode rapidamente regredir para imaturidade em gestão de projetos. O conhecimento é perdido e os erros do passado se repetem" (KERZNER, 2002, p. 52).

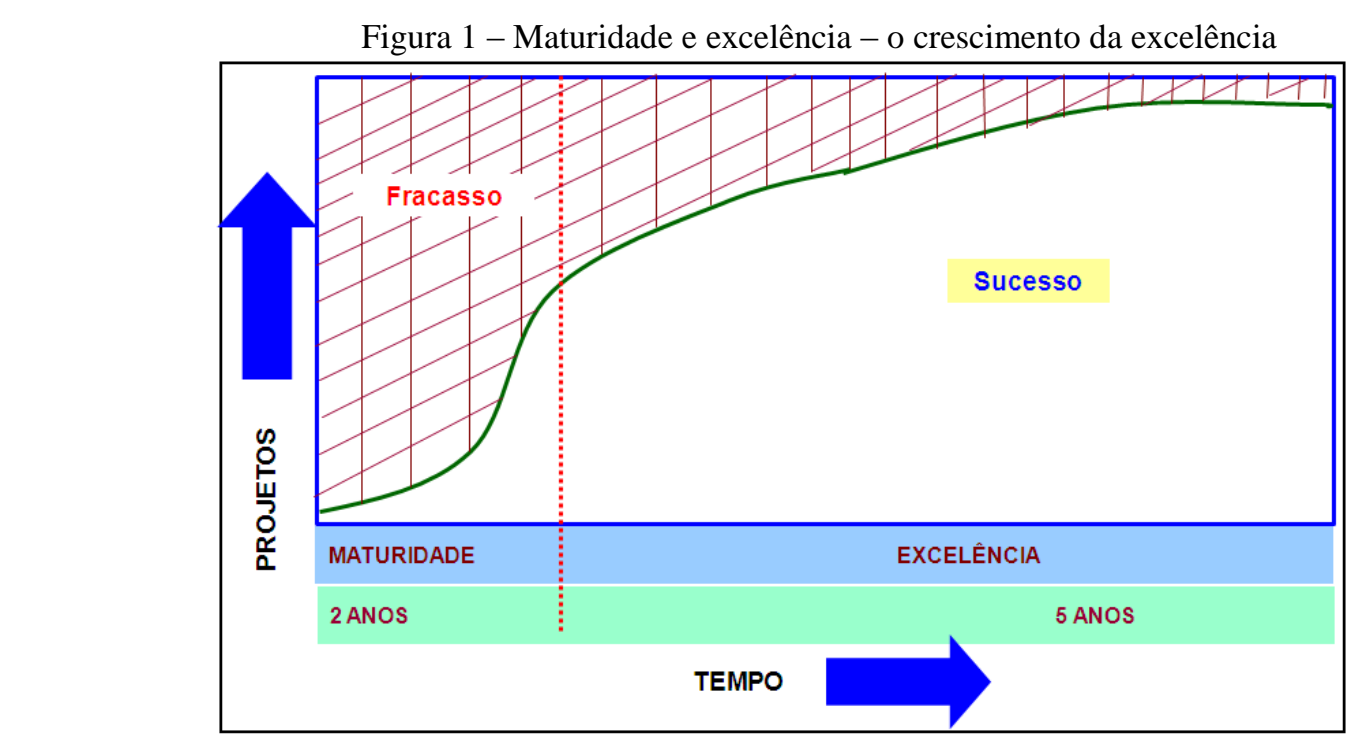

Fonte: adaptada de Kerzner (2009, p.59)

Em relação às lições aprendidas, segundo Baumotte (2009) trata-se de avaliar erros e acertos nos projetos, identificando o que poderia ter sido evitado e o que poderá ser feito diferente e/o melhor da próxima vez. Esse entendimento é endossado por Valeriano (2005), que as considera uma 
das formas mais eficientes para se obter e ampliar o conhecimento na área de gerenciamento de projetos.

\subsection{Modelos de maturidade em gerenciamento de projetos}

Os modelos de maturidade em gerenciamento de projetos (MMGP) são utilizados para realizar uma avaliação da capacidade de uma organização executar projetos com sucesso, tendo como base boas práticas preestabelecidas. Ademais, os MMGP permitem às organizações identificarem onde elas precisam melhorar no gerenciamento de projeto e, consequentemente, estabelecerem planos de desenvolvimento de sua maturidade na execução dos empreendimentos, além de servirem de referência para compararem seu nível de maturidade com o de outras companhias (PRADO, 2010; HERKENHOFF, FIGUEIREDO e LIMA, 2011; IBBS e KWAK, 2000; ANDERSEN e JESSEN, 2003; RABECHINI, 2005; LUKOSEVICIUS e CAMPOS FILHO, 2008, VERGOPIA, 2008).

Existem vários modelos de maturidade e não há um consenso ou padrão para avaliá-la. Os diversos modelos existentes apresentam diferenças em suas características, em seus fatores, e na abordagem para avaliar a maturidade (KHOSHGOFTAR e OSMAN, 2009; VERGOPIA, 2008; RABECHINI, 2005; IBBS e KWAK, 2000).

\subsection{Método KPMMM ${ }^{\circledR}$}

O método KPMMM ${ }^{\circledR}$ foi proposta por Kerzner em 1999, ajustada em 2001 e em 2005 com publicação de livros específicos para divulgá-la. Esse modelo, assim como a abordagem do $\mathrm{CMM}^{\circledR}$ revisto e renomeado para $\mathrm{CMMI}^{\circledR}$, propõe cinco níveis de maturidade com conceitos similares (CARVALHO et al, 2005; RABECHINI e PESSÔA, 2005; KERZNER, 2005; RABECHINI, 2012).

De acordo com Carvalho e Rabechini (2008), a abordagem KPMMM ${ }^{\circledR}$ de Kerzner (2005) foi originalmente desenvolvida em 2000 e 2001, a partir dos esforços de Kerzner para adaptar o modelo $\mathrm{CMM}^{\circledR}$ para a gestão de projetos.

Na Figura 2 apresenta-se um mapa do método KPMMM $^{\circledR}$ de Kerzner (2005), que complementa e apresenta uma síntese das informações do Quadro 2.

A síntese dos cinco níveis do modelo $\mathrm{KPMMM}^{\circledR}$ são (KERZNER 2005):

a) nível 1 - linguagem comum: nesse nível a organização reconhece a contribuição da abordagem de gerenciamento e a necessidade de desenvolver um bom entendimento em relação à abordagem de gerenciamento de projeto, estabelecendo uma terminologia comum; 
b) nível 2 - processos comuns: é atingido quando a organização reconhece a necessidade de definir, desenvolver e estabelecer processos comuns para que o sucesso se repita em diversos projetos. Também pertencem a esse nível o reconhecimento e apoio para utilização dos princípios de gerenciamento de projetos em outros métodos aplicados pela organização;

c) nível 3 - método singular: atinge-se esse nível quando a organização reconhece que a combinação em uma única método de todas as métodos corporativas utilizadas possibilita um efeito sinérgico. A abordagem de gerenciamento de projetos é a método central desse processo de combinação;

d) nível 4 - benchmarking: consiste na realização de um processo contínuo de comparação das práticas de gerenciamento de projetos desenvolvidas pela companhia com outras organizações. Essa abordagem torna-se fonte de sustentação de um processo contínuo de melhoria de seu desempenho, necessário para sustentar a vantagem competitiva; e

e) nível 5 - melhoria contínua: nesse nível as organizações avaliam o conhecimento adquirido com aplicação do benchmarking (nível 4) para identificar e definir o que será incorporado à método da organização para seu aprimoramento.

Ao analisar a Figura 2 observa-se que as organizações nos níveis 1 e 2 são classificadas como imaturas; as do nível 3 como maduras; e que as que atingem os níveis 4 e 5 enquadram-se na excelência (KERZNER 2005, KERZNER 2002; CARVALHO et al., 2005; RABECHINI, 2005 e CARVALHO e RABECHINI, 2008).

Para cada nível a método $\mathrm{KPMMM}^{\circledR}$ propõe uma abordagem específica para avaliar o nível de maturidade em gerenciamento de projetos. As diretrizes para avaliação estão apresentadas no Quadro 2, o qual complementa a Figura 2.

Ao analisar o Quadro 2 e Figura 2 observa-se que a método KPMMM ${ }^{\circledR}$ propõe cinco níveis para avaliação da maturidade da organização, que pode situar-se em qualquer ponto no contínuo. Para os cinco níveis propõe-se como principal instrumento de avaliação a realização de questionários (KERZNER, 2005).

Observa-se que no nível 1 realiza-se uma prova, com questões em que se busca aferir se os profissionais conhecem os conceitos básicos de gerenciamento de projeto. Nessa avaliação a método $\mathrm{KPMMM}^{\circledR}$, de Kerzner (2005), toma como base o PMBOK Guide do PMI ${ }^{\circledR}$ (2008a), publicado pelo $\mathrm{PMI}^{\circledR}$. Na avaliação dos níveis 2; 4; e 5 são apresentadas declarações para que os profissionais indiquem sua percepção de quanto desenvolvidas estão as características de cada nível na organização analisada (KERZNER, 2005). 
A técnica de avaliação do nível 3 difere das dos níveis 2, 4 e 5, por não apresentar escalas de -3 (discorda totalmente) a +3 (concorda totalmente) para capturar a percepção, e sim de 04 a 05 alternativas para cada questão (KERZNER, 2005).

O método KPMMM ${ }^{\circledR}$ de Kerzner (2005), além de utilizar o PMBOK Guide do PMI $^{\circledR}$ (2008a) como referência, também integra o conceito de Project Management Office (PMO) no nível estratégico (CARVALHO et al, 2005; RABECHINI, 2005 e CARVALHO e RABECHINI, 2008).

As organizações podem avançar em dois níveis simultaneamente. Os níveis 1 e 2 são sobrepostos, assim como os níveis 3; 4 e 5. É importante ressaltar que segundo a método $\mathrm{KPMMM}^{\circledR}$ de Kerzner (2005) os níveis 3, 4 e 5 formam um ciclo contínuo de feedback, por meio do qual as organizações podem alcançar e sustentar a excelência em gerenciamento de projetos (LUKOSEVICIUS e CAMPOS FILHO, 2008; KERZENER, 2005).

Ao observar-se a Figura 3 identifica-se no nível 2 a representação do ciclo de vida genérico para maturidade em gerenciamento de projetos, que segundo o modelo KPMMM $^{\circledR}$ de Kerzner (2005) é composto por cinco fases (CARVALHO et al, 2005; SEGISMUNDO e CARVALHO, 2006; KERZNER, 2005 e CARVALHO e RABECHINI, 2008):

a) Embrionária: ocorre com o reconhecimento ou aceitação da importância ou benefícios do gerenciamento de projetos para a organização;

b) Reconhecimento/aceitação da alta administração: quando se observa na companhia visibilidade em termos de suporte dos executivos, entre outros; compreensão pela gerência do gerenciamento de projetos; presença de um patrocinador da alta administração; e adoção de uma posição favorável à realização de mudanças na maneira como a entidade faz os negócios;

c) Reconhecimento ou aceitação da média gerência: caracteriza-se pelo envolvimento visível da média gerência; compromisso dos gerentes com os objetivos; liberação de recursos necessários à execução dos projetos; e participação de treinamentos em método de gerenciamento de projetos;

d) Crescimento: ocorre com o início da criação de um processo de gerenciamento de projetos e compromisso em realizar e colocar em prática o planejamento; e

e) Maturidade: essa fase se caracteriza pelo início do desenvolvimento formal de um sistema integrado para controlar custo e cronograma, conjuntamente com o desenvolvimento de um programa de educação continuada para suportar e ampliar as competências dos profissionais envolvidos com gerenciamento de projetos.

Para o nível 3, a método $\mathrm{KPMMM}^{\circledR}$ de Kerzner (2005) agrega as seis características do nível três para compor o denominado Hexágono da Excelência. O desenvolvimento dos seis traços ou 
áreas identificadas nesse nível é que irão diferenciar as entidades excelentes em gerenciamento de projetos das demais organizações com desempenho médio (KERZNER, 2005).

O processo de benchmarking é a característica chave do nível 4. Deve ser realizado de maneira contínua, pela comparação das práticas de gerenciamento de projetos com as práticas dos líderes em qualquer lugar do mundo. Esse processo traz informações e insights para ajudar aprimorar o desempenho da organização (KERZNER, 2005). 
Quadro 2 - Panorama conceitual do método KPMMM ${ }^{\circledR}$

\begin{tabular}{|c|c|c|c|c|c|}
\hline $\begin{array}{c}\text { Níve } \\
\text { l }\end{array}$ & Descrição & \begin{tabular}{|c|} 
Grau de \\
Dificuldade
\end{tabular} & Barreira ao Nível & Características do Nível & Instrumento de Avaliação do Nível \\
\hline 1 & $\begin{array}{l}\text { Linguagem } \\
\text { Comum }\end{array}$ & Médio & $\begin{array}{l}\text { a) Resistência à mudança. } \\
\text { b) Deixar-se suficiente bem } \\
\text { sozinho. } \\
\text { c) Não inventado aqui. } \\
\text { d) Isso não se aplica para nós. } \\
\text { e) Nós não precisamos disso. }\end{array}$ & $\begin{array}{l}\text { a) Linguagem comum para } \\
\text { Gerenciamento de Projetos. } \\
\text { b) Não suporte do nível executivo. } \\
\text { c) Não há tentativa para reconhecer os } \\
\text { benefícios do gerenciamento de } \\
\text { projetos. } \\
\text { d) Auto interesses vem antes dos } \\
\text { interesses da companhia. } \\
\text { e) Não há investimento em treinamento e } \\
\text { educação para os gerentes de projetos. }\end{array}$ & $\begin{array}{l}\text { Critério de Avaliação: possuir conhecimento sobre os } \\
\text { fundamentos e princípios do gerenciamento de projetos. Uma boa } \\
\text { compreensão do PMBOK Guide é suficiente para completar o nível } \\
\text { 1. } \\
\text { Método de Avaliação: } 80 \text { questões baseadas no Guia PMBOK, } \\
\text { subdividas em oito categorias: gerenciamento do escopo; custo; } \\
\text { recursos humanos; compras, qualidade; risco e comunicação. } \\
\text { Escala de Avaliação: seis ou mais questões corretas em cada uma } \\
\text { das categorias indicam que o nível } 1 \text { foi completado de forma } \\
\text { satisfatória. }\end{array}$ \\
\hline 2 & $\begin{array}{c}\text { Processos } \\
\text { Comuns }\end{array}$ & Médio & $\begin{array}{l}\text { a) Resistência ao novo método. } \\
\text { b) Nós já realizamos o trabalho } \\
\text { bem. } \\
\text { c) Acreditar que a método precisa } \\
\text { ser formada por políticas e } \\
\text { procedimentos rígidos. } \\
\text { d) Resistência para } \\
\text { responsabilidade horizontal. }\end{array}$ & $\begin{array}{l}\text { a) Reconhecimento dos benefícios do } \\
\text { gerenciamento de projetos. } \\
\text { b) Suporte da organização em todos os } \\
\text { níveis. } \\
\text { c) Reconhecimento da necessidade } \\
\text { processo/método. } \\
\text { d) Reconhecimento da necessidade de } \\
\text { processo para controle de custos. } \\
\text { e) Desenvolvimento de Currículo para } \\
\text { treinamento dos gerentes de projetos. }\end{array}$ & $\begin{array}{l}\text { Critério de avaliação - são quatro: } \\
\text { a) Desenvolvimento de uma cultura que suporte o GP. } \\
\text { b) Reconhecimento das forças, necessidades e benefícios em curto } \\
\text { e longo termo (ambos). } \\
\text { c) Desenvolvimento de um processo/método. } \\
\text { d) Desenvolvimento de programa de formação contínua. } \\
\text { Método de Avaliação: } 20 \text { declarações para avaliar as cinco fases } \\
\text { do ciclo de vida. Cada questão possui uma escala de }-3 \text { a }+3 \text { para } \\
\text { avaliar de total desacordo a total acordo com a declaração. As } \\
\text { declarações são subdivididas em quatro para avaliar cada fase do } \\
\text { ciclo de vida. } \\
\text { Escala de Avaliação: seis ou mais pontos em cada fase do ciclo de } \\
\text { vida indicam se o nível foi completado de forma satisfatória. }\end{array}$ \\
\hline 3 & $\begin{array}{l}\text { Método } \\
\text { Singular }\end{array}$ & Alto & $\begin{array}{l}\text { a) Não mexa se ele não está } \\
\text { quebrado. } \\
\text { b) Resistência para um método } \\
\text { singular (isto é, processos } \\
\text { repetidos). } \\
\text { c) Resistência em compartilhar } \\
\text { responsabilidade. } \\
\text { d) Cultura corporativa } \\
\text { fragmentada. } \\
\text { e) Excessiva ênfase em } \\
\text { documentar. }\end{array}$ & $\begin{array}{l}\text { a) Processos integrados. } \\
\text { b) Cultura de suporte. } \\
\text { c) Suporte gerencial em todos os níveis. } \\
\text { d) Gerenciamento de projetos informal. } \\
\text { e) Retorno do investimento para o } \\
\text { treinamento em gerenciamento de } \\
\text { projetos. } \\
\text { f) Comportamento de excelência. }\end{array}$ & $\begin{array}{l}\text { Critério de avaliação: as seis características. } \\
\text { Método de Avaliação: } 42 \text { questões/declarações são dividas em seis } \\
\text { conjuntos para avaliar as seis características. } \\
\text { Escala de Avaliação: quatro intervalos de pontuação (169-210); } \\
\text { (147-168); (80-146) e menor de } 80 \text {. O nível quatro é satisfatório } \\
\text { quando a que } 169 .\end{array}$ \\
\hline
\end{tabular}




\begin{tabular}{|c|c|c|c|c|c|}
\hline 4 & $\begin{array}{c}\text { Benchmarki } \\
n g\end{array}$ & Baixo & $\begin{array}{l}\text { a) Síndrome do não inventado } \\
\text { aqui. } \\
\text { b) Não se aplica para nós. } \\
\text { c) Indústria errada para realizar } \\
\text { benchmarking. } \\
\text { d) Temor de quais resultados irão } \\
\text { ser encontrados. } \\
\text { e) Resistência para mudança. }\end{array}$ & $\begin{array}{l}\text { a) Estabelecimento de um Escritório de } \\
\text { Projetos (EP) ou Project Office (PO). } \\
\text { b) Dedicação para benchmarking. } \\
\text { c) Olhar para similar e não similares } \\
\text { indústrias. } \\
\text { d) Benchmarking quantitativo: processos e } \\
\text { método. } \\
\text { e) Benchmarking qualitativo: culturas. }\end{array}$ & $\begin{array}{l}\text { Critério de avaliação: são quatro. } \\
\text { a) Criar uma organização dedicada para benchmarking. } \\
\text { b) Desenvolver um processo de benchmarking para gerenciamento } \\
\text { de projetos. } \\
\text { c) Decidir sobre o que e contra quem o benchmarking será } \\
\text { realizado. } \\
\text { d) Reconhecer os benefícios do benchmarking. } \\
\text { Método de Avaliação: } 25 \text { declarações para avaliar os dois tipos de } \\
\text { benchmarking. Cada questão possui uma escala de }-3 \text { a }+3 \text { para } \\
\text { avaliar total desacordo a total acordo com a declaração. As } \\
\text { declarações são subdivididas em dois grupos uma para } \\
\text { benchmarking quantitativo (15 questões) e qualitativo (10 } \\
\text { questões). } \\
\text { Escala de Avaliação: nota maior que } 25 \text {, a organização completou } \\
\text { o nível; nota menor do que } 10 \text {, ainda não compreendeu o } \\
\text { benchmarking e sua importância e nota entre } 11 \text { a } 24 \text {, a organização } \\
\text { está a caminho de atingir o nível. }\end{array}$ \\
\hline 5 & $\begin{array}{l}\text { Melhoria } \\
\text { Contínua }\end{array}$ & Baixo & $\begin{array}{l}\text { Não se identificou de forma } \\
\text { explícita, mas os principais } \\
\text { parecem ser: } \\
\text { a) Falta de apoio da alta } \\
\text { administração; } \\
\text { b) Falta de compromisso de toda a } \\
\text { organização com o } \\
\text { benchmarking e melhoria } \\
\text { contínua; } \\
\text { c) Processo excessivamente } \\
\text { formal e burocrático. }\end{array}$ & $\begin{array}{l}\text { a) Arquivo de lições apreendidas. } \\
\text { b) Transferência de conhecimento. } \\
\text { c) Programa de mentoring. } \\
\text { d) Planejamento estratégico para } \\
\text { gerenciamento de projetos. }\end{array}$ & $\begin{array}{l}\text { Critério de avaliação: os gerentes de projetos são considerados } \\
\text { profissionais e existe um plano de carreira com descrição de cargos } \\
\text { e responsabilidades. } \\
\text { Método de Avaliação: } 16 \text { declarações para avaliar esse nível sem } \\
\text { subdivisões, por meio de uma escala de }-3 \text { a +3 para avaliar a total } \\
\text { desacordo a total acordo com a declaração. } \\
\text { Escala de Avaliação: categorizada em três: } \\
\text { a) igual ou maior do que } 20 \text { pontos a organização está } \\
\text { comprometida com benchmarking e melhoria contínua. A } \\
\text { organização apresenta um padrão de excelência. } \\
\text { b) entre } 10 \text { e } 19 \text { pontos indicam que a organização iniciou processo } \\
\text { de melhoria contínua, mas precisa acelerá-lo. Existe hesitação. A } \\
\text { organização está a caminho da excelência. } \\
\text { c) Nota menor do que } 10 \text { indica uma forte resistência ou falta de } \\
\text { suporte da alta administração para suportar a melhoria contínua. A } \\
\text { organização ainda não iniciou de fato o caminho para atingir a } \\
\text { excelência. }\end{array}$ \\
\hline
\end{tabular}

Fonte: adaptado de Kerzner (2005) 
Figura 3 - Mapa do método KPMMM ${ }^{\circledR}$

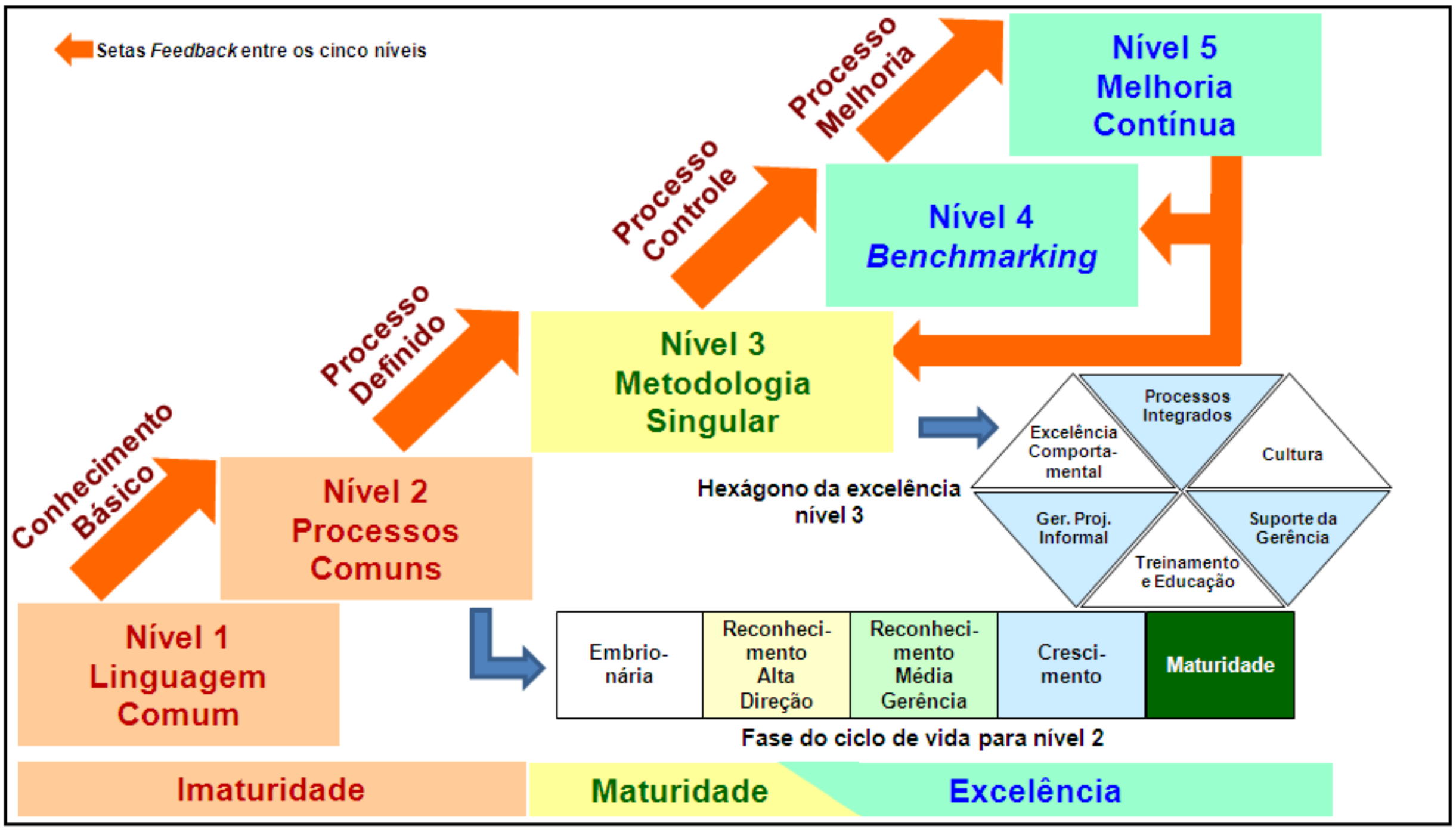

Fonte: adaptada de Kerzner (2005 e 2009); Carvalho et al. (2005) e Carvalho e Rabechini (2008) 
No nível 5 a melhoria contínua deve ser buscada em cinco dimensões da abordagem do gerenciamento de projetos (KERZNER, 2005): primeira: processos existentes para gerenciamento de projetos; segunda: integração dos processos; terceira: gerenciamento de questões ou problemas; quarta: benchmarking; e quinta: padrões de comportamento.

\subsection{Método OPM3 ${ }^{\circledR}$}

O método OPM3 ${ }^{\circledR}$ é fruto de uma iniciativa do PMI ${ }^{\circledR}$ e foi iniciada em 1998 para desenvolver um modelo de maturidade organizacional em gerenciamento de projetos. Após vários anos de trabalho envolvendo centenas de profissionais de diversos países, dentre eles alguns voluntários, foi lançada pelo PMI ${ }^{\circledR}$ em dezembro de 2003 a primeira versão do modelo $\mathrm{OPM}^{\circledR}\left(\mathrm{PMI}^{\circledR}, 2008\right)$.

Em 2004 o PMI $^{\circledR}$ formou e designou um novo grupo de profissionais com a missão de realizar uma revisão das lições aprendidas com uso da abordagem e propor ajustes/melhorias na primeira versão. Como resultado dessa missão em 2008 foi lançado a $2^{\mathrm{a}}$ edição (PRADO, 2010; PMI ${ }^{\circledR}, 2008 ;$ ARCHIBALD, 2005 e SCHLICHTER, 2001).

$\mathrm{O}$ modelo do método OPM3 ${ }^{\circledR}$ do $\mathrm{PMI}^{\circledR}$ (2008) para avaliação da maturidade em gerenciamento de projetos estruturou a análise em três constructos, domínios ou dimensões: projetos, programas, portfólios. Para cada uma dessas dimensões são definidos quatro estágios de maturidade dentro do grupo de processo para cada domínio, alinhados com os objetivos estratégicos (PMI ${ }^{\circledR}, 2008$; CARVALHO et al, 2005; CARVALHO e RABECHINI, 2008).

Além desses três constructos principais existe um quarto, denominado organização do gerenciamento do projeto, que cobre as competências da organização para executar os outros três constructos (projeto, programa e portfólio) e coordena o alinhamento com o planejamento estratégico da organização (PMI ${ }^{\circledR}, 2008$ e PRADO, 2010). Um resumo dos termos e conceitos principais para interpretação do modelo OPM $3{ }^{\circledR}$ do PMI ${ }^{\circledR}$ (2008) apresenta-se no Quadro 3.

Além dos termos apresentados no Quadro 3 existem dois conceitos importantes para compreender a abordagem do OPM $3^{\circledR}$ do PMI $^{\circledR}$ (2008), os organizacionais e a maturidade, que fazem parte do nome da método. Dentro do referencial teórico do OPM3 ${ }^{\circledR}$ o conceito organizacional refere-se à ampliação do escopo do modelo de gestão de projetos, que extrapola o conceito de projeto em si, foco do PMBOK Guide do $\mathrm{PMI}^{\circledR}$ (2008a), para cobrir toda a organização (PMI $\left.{ }^{\circledR}, 2008\right)$. 
Quadro 3 - Principais conceitos do método OPM3 ${ }^{\circledR}$

\begin{tabular}{|c|c|c|c|c|}
\hline Termo Inglês & $\begin{array}{l}\text { Sigla } \\
\text { Inglês }\end{array}$ & $\begin{array}{l}\text { Tradução } \\
\text { Adotada }\end{array}$ & $\begin{array}{c}\text { Sigla } \\
\text { Português }\end{array}$ & Conceito \\
\hline $\begin{array}{l}\text { Organizational } \\
\text { Project } \\
\text { Management }\end{array}$ & OPM & $\begin{array}{l}\text { Organização do } \\
\text { Gerenciamento } \\
\text { de Projetos }\end{array}$ & OGP & $\begin{array}{l}\text { É o gerenciamento sistemático de projetos, } \\
\text { programas e portfólios alinhados com os } \\
\text { objetivos estratégicos da organização. }\end{array}$ \\
\hline Organization & - & Organização & - & $\begin{array}{l}\text { Pode referir-se a uma companhia ou negócio, } \\
\text { agência, associação, ou sociedade, grupo, } \\
\text { departamento, ou qualquer outra subunidade } \\
\text { dentro uma organização. }\end{array}$ \\
\hline $\begin{array}{l}\text { Project } \\
\text { Management } \\
\text { Domain } \\
\end{array}$ & - & $\begin{array}{l}\text { Domínios do } \\
\text { Gerenciamento } \\
\text { de Projetos }\end{array}$ & - & $\begin{array}{l}\text { Descreve as áreas de conhecimento e os grupos } \\
\text { de processo que guiam a execução de projetos } \\
\text { individuais. }\end{array}$ \\
\hline Constructo & - & Constructo & - & $\begin{array}{l}\text { OPM3 utiliza os constructos de Gerenciamento } \\
\text { de: Projeto, Programa e Portfólio para } \\
\text { representar a escala de sofisticação e controle. }\end{array}$ \\
\hline $\begin{array}{l}\text { Organization } \\
\text { Enablers }\end{array}$ & $\mathrm{OE}$ & $\begin{array}{l}\text { Capacidades } \\
\text { Organizacio- } \\
\text { nais }\end{array}$ & $\mathrm{CO}$ & $\begin{array}{l}\text { As capacidades organizacionais são as } \\
\text { Melhores Práticas que facilitam a } \\
\text { implementação de Melhores Práticas, mas } \\
\text { também ajudam a fazer a melhoria } \\
\text { organizacional ser sustentável. }\end{array}$ \\
\hline Capability & - & Competências & - & $\begin{array}{l}\text { São as competências que devem existir na } \\
\text { organização para executar os processos e } \\
\text { entregar os resultados estabelecidos pelo OPM. }\end{array}$ \\
\hline $\begin{array}{l}\text { Key } \\
\text { Perfomance } \\
\text { Indicator }\end{array}$ & KPI & $\begin{array}{l}\text { Indicador Chave } \\
\text { de Desempenho }\end{array}$ & ICD & $\begin{array}{l}\text { É um critério pelo qual uma organização pode } \\
\text { determinar, quantitativamente ou } \\
\text { qualitativamente, se uma entrega ou resultado } \\
\text { existe e em que grau. }\end{array}$ \\
\hline
\end{tabular}

Fonte: adaptado de $\left.\mathrm{PMI}^{(}\right)(2008)$

Essa ampliação desloca o foco de operacional, execução de projetos, para o estratégico, por essa razão o modelo incorpora, além de projetos, programas e portfólios, e a promoção do alinhamento dos projetos à estratégia do negócio. Por outro lado, o conceito de maturidade abarca as melhores práticas e capacitações que a organização possui e as que necessitam ser desenvolvidas para sustentar e aprimorar uma cultura para que os projetos sejam executados com sucesso e alinhados aos objetivos definidos no planejamento estratégico $\left(\mathrm{PMI}^{\circledR}, 2008\right)$. Para complementar as explanações, a Figura 4 apresenta o mapa do modelo OPM $3^{\circledR}$.

Ao observar-se a Figura 3 constata-se que o modelo OPM $3{ }^{\circledR}$ do PMI ${ }^{\circledR}(2008)$ avalia a maturidade pelo ciclo de estágios ou níveis que vai da padronização até a melhoria contínua, indicado pelas setas no sentido horizontal. Essa avaliação pode ser realizada para cada um dos constructos - projeto, programa e portfólios - e/ou dos grupos de processo de cada um dos constructos - projeto, programa e portfólio, utilizando-se para avaliação de cada um dos elementos citados as normas divulgadas pelo PMI ${ }^{\circledR}\left(\mathrm{PMI}^{\circledR}, 2008\right.$; PRADO 2010). 
Figura 3 - Mapa do método OPM3 ${ }^{\circledR}$

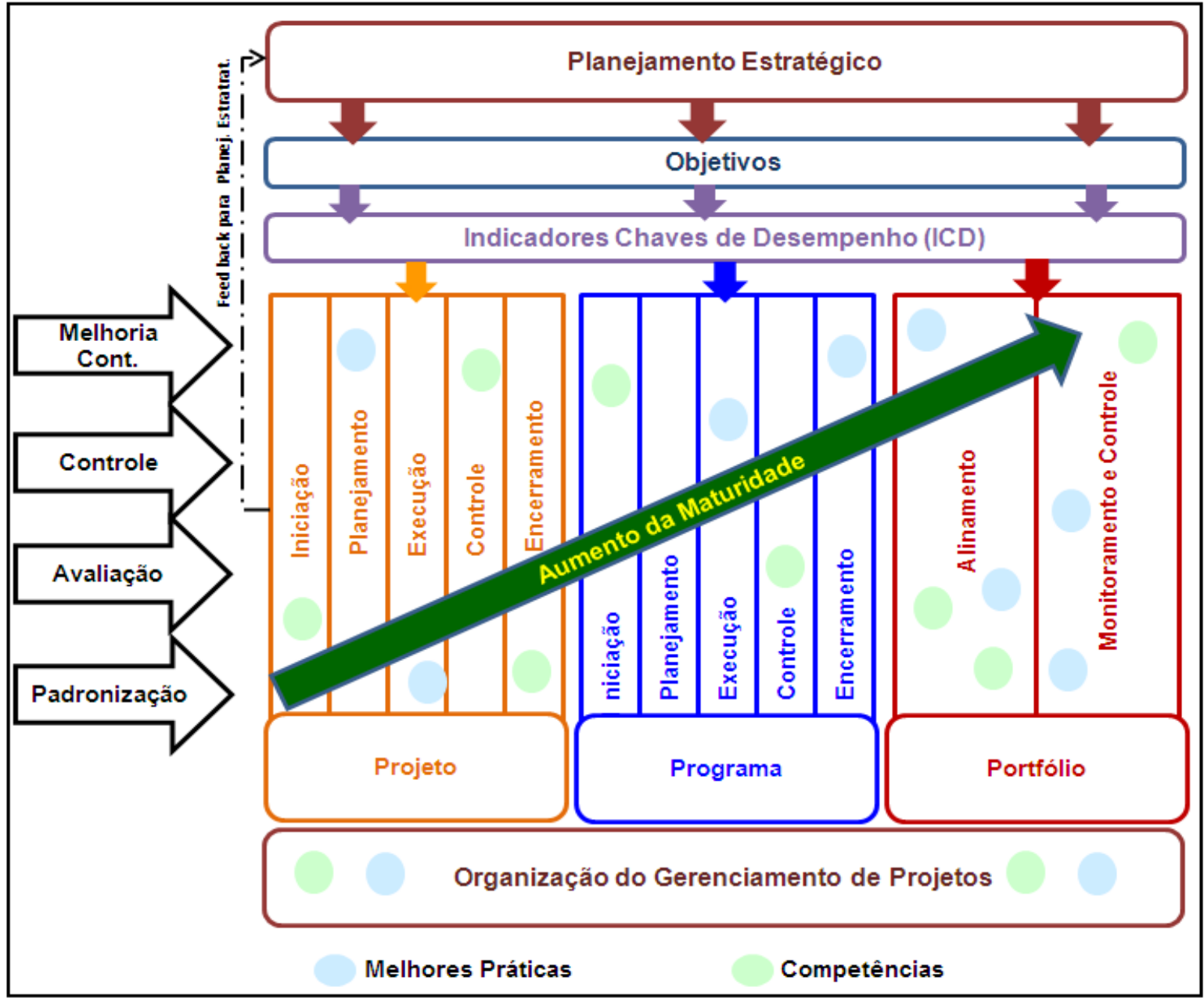

Fonte: adaptada de $\mathrm{PMI}^{\circledR}(2008)$

A maturidade dentro do referencial teórico do $\mathrm{OPM} 3^{\circledR}$ do $\mathrm{PMI}^{\circledR}$ (2008) implica, portanto, na melhoria contínua das competências organizacionais com vista a atingir a excelência organizacional na execução dos projetos (PRADO, 2010; CARVALHO e RABECHINI, 2005).

Segundo essa abordagem, as melhores práticas, representadas na Figura 4 por círculos azuis, e as competências, representadas por círculos verdes, estão difusas pela organização. A método OPM $3^{\circledR}$ do PMI ${ }^{\circledR}$ (2008), também se propõe a identificar quais melhores práticas ou melhorias a organização precisa implementar ou aprimorar (PMI ${ }^{\circledR}, 2008$; PRADO, 2010).

Para realizar a avaliação da maturidade, a método OPM $3^{\circledR}$ do PMI ${ }^{\circledR}$ (2008), tem um questionário de 125 questões que permitem uma auto avaliação do nível de maturidade da organização em todos os constructos: projeto, programa e portfólios, apresentados na Figura 4. Após essa auto avaliação é possível, por meio da utilização de um banco de dados com mais de 600 melhores práticas catalogadas por constructo e/ou grupo de processo, definir um plano de ação para elevar o nível de maturidade da organização (PMI ${ }^{\circledR}, 2008$; PRADO 2010). 
Uma vez finalizada a execução dos planos de ação, uma nova rodada de avaliação deve ser realizada. Esse ciclo de análise do conhecimento (melhores práticas), avaliação e execução de um plano de ação para melhoria pode ser executado continuamente para toda a organização ou somente para uma parte, grupo de processo específico ou um constructo, de acordo com as prioridades da organização (PMI ${ }^{\circledR}$, 2008; PRADO 2010).

O resultado das ações de melhoria é avaliado pelo ICD (KPI) e os ajustes são implementados conforme são identificadas as necessidades. Em resumo, a método OPM $3^{\circledR}$ do $\mathrm{PMI}^{\circledR}$ (2008) permite avaliar a maturidade organizacional em gerenciamento de projetos: domínios, dimensões, grupos de processos de gerenciamento de projetos e estágios de melhoria (PRADO, 2010; PMI ${ }^{\circledR}$, 2008; CARVALHO e RABECHINI, 2005; LUKOSEVICIUS e CAMPOS FILHO, 2008).

Essa possibilidade de avaliar a maturidade em diversas visões possibilita às organizações flexibilidade e suporte no processo de tomadas de decisão, assim como na definição e planejamento das melhorias a serem incorporadas. Além disso, o PMI ${ }^{\circledR}$ prove treinamento, suporte, software e material para aplicação do método OPM3 ${ }^{\circledR}$ para as organizações que se interessam (PRADO, 2010; PMI ${ }^{\circledR}, 2008$; CARVALHO e RABECHINI, 2005; LUKOSEVICIUS e CAMPOS FILHO, 2008).

\section{Método e procedimentos}

As pesquisas podem ser classificadas segundo diferentes tipologias. As classificações variam em função de quem as elabora, e diversos fatores influenciam a divisão dos tipos (MARCONI e LAKATOS, 2011).

Segundo a classificação proposta por Silva e Menezes (2005, p. 20), "este estudo, do ponto de vista de sua natureza é uma pesquisa aplicada", pois envolve o estudo de um método para aplicação prática. Com relação à sua abordagem é qualitativa, busca realizar a interpretação de dois métodos e compreender sua aplicação.

\section{Resultados e discussão}

Nessa seção é apresentada, analisada e discutida, com base na revisão de literatura, os resultados da pesquisa bibliográfica, para atingir o objetivo proposto: analisar e comparar os métodos OPM3 ${ }^{\circledR}$ e $\mathrm{KPMMM}^{\circledR}$ para avaliação de maturidade de gerenciamento de projetos com base em pesquisa bibliográfica. 
Nesta seção apresentam-se os resultados das pesquisas e reflexões realizadas com base na literatura examinada sobre o tema.

\subsection{Análise das abordagens $\mathrm{OPM3}^{\circledR} \mathrm{E}^{\mathrm{KPMM}} \mathrm{M}^{\circledR}$}

Conforme observado por Herkenhoff, Figueiredo e Lima (2011), uma diferença que dificulta a comparação dos modelos de avaliação de maturidade é o fato de o método OPM3 ${ }^{\circledR}$ apresentar quatro níveis, enquanto que a $\mathrm{KPMMM}^{\circledR}$ apresenta cinco.

Um resumo com as principais características dos métodos $\mathrm{OPM} 3^{\circledR}$ e $\mathrm{KPMMM}^{\circledR}$ com base em Cirilo (2010) é apresentado no Quadro 4.

A partir da análise do Quadro 4 percebe-se que as duas métodos apresentam três diferenças importantes em relação às características principais:

a) $\mathbf{1}^{\mathbf{a}}$ em relação à origem: a $\mathrm{KPMMM}^{\circledR}$ foi criada por um pesquisador enquanto a $\mathrm{OPM} 3{ }^{\circledR}$ é fruto do trabalho de uma entidade;

b) $2^{\mathrm{a}}$ em relação ao fundamento para avaliação: a $\mathrm{KPMMM}^{\circledR}$ utiliza o $P M B O K^{\circledR}$ Guide (PMI ${ }^{\circledR}$ 2008a) enquanto a $\mathrm{OPM}^{\circledR}$ se baseia num banco de dados das melhores práticas;

c) $3^{\mathbf{a}} \mathbf{e m}$ relação aos níveis: enquanto a $K P M M M^{\circledR}$ classifica a maturidade em cinco níveis a OPM $3^{\circledR}$ a classifica em quatro.

Quadro 4 - Comparação principais características OPM $3^{\circledR}$ e $\mathrm{KPMMM}^{\circledR}$

\begin{tabular}{|l|l|l|}
\hline \multicolumn{1}{|c|}{ Nome } & \multicolumn{1}{|c|}{$\begin{array}{c}\text { Kerzner Project Manager Maturity } \\
\text { Model }\end{array}$} & $\begin{array}{c}\text { Organizational Project } \\
\text { Management Maturity Model }\end{array}$ \\
\hline Sigla & KPMMM $^{\circledR}$ & OPM3 ${ }^{\circledR}$ \\
\hline Origem & Desenvolvido por Kerzner & Desenvolvido pelo PMI ${ }^{\circledR}$ \\
\hline Fundamento & Alinhado com PMBOK & Alinhado com melhores práticas \\
\hline Níveis & Abrange cinco níveis de maturidade & $\begin{array}{l}\text { Abrange quatro níveis de } \\
\text { maturidade }\end{array}$ \\
\hline Abordagem & 183 questões & 125 questões sim ou não \\
\hline Realização & Realizado papel ou on line & Realizado papel ou on line \\
\hline Aplicação & Qualquer tipo de empresa & Qualquer tipo de empresa \\
\hline
\end{tabular}

Fonte: adaptado de Cirilo (2010)

Quanto aos níveis de maturidade, segundo Herknhof, Figueiredo e Lima (2011), os níveis 1 e 2 do modelo OPM ${ }^{\circledR}{ }^{\circledR}$ exigem o atendimento a critérios mais avançados do que o modelo $\mathrm{KPMMM}^{\circledR}$. Já o nível 3 do OPM3 ${ }^{\circledR}$ assemelha-se, em termos de requerimento, ao nível 4 e 5 do modelo $\mathrm{KPMMM}^{\circledR}$. Essa análise parece ser corroborada pelo estudo realizado por Carvalho et al (2005), que indica existir complementaridade entre os dois modelos no que 
concerne ao resultado da avaliação. Apesar de apresentarem diferenças, ambos têm foco na institucionalização do gerenciamento de projeto.

Outra diferença relevante entre os dois modelos é o fato de o OPM $3{ }^{\circledR}$ trabalhar com o conceito de dimensões - projetos, programa e portfólio - que não estão presentes na abordagem do KPMMM ${ }^{\circledR}$. Por outro lado, essa método permite sobreposição entre os níveis e feedback, num ciclo repetitivo entre os níveis 3, 4 e 5 (LUKOSEVICIUS; CAMPOS FILHO, 2008).

As discussões anteriores, realizadas, podem ser complementadas com a observação de que a método KPMMM ${ }^{\circledR}$ apresenta o conceito de ciclo de vida para o nível 2 e o hexágono da excelência para o nível 3. Por outro lado, a método OPM $3^{\circledR}$ considera, além do conceito de dimensões, as competências organizacionais, o grupo de processos, e as melhores práticas, ampliando os fatores que diferenciam ambas.

No Quadro 5 apresenta-se um resumo comparativo das características presentes, da aplicação e limitações das abordagens $\mathrm{OPM} 3^{\circledR}$ e $\mathrm{KPMMM}^{\circledR}$, analisadas nos parágrafos anteriores.

Quadro 5 - Aplicação e limitações dos métodos OPM3 ${ }^{\circledR}$ e $\mathrm{KPMMM}^{\circledR}$

\begin{tabular}{|c|c|c|}
\hline Aplicação & KРММM $^{\circledR}$ & $\mathrm{OPM}^{\circledR}{ }^{\circledR}$ \\
\hline Avaliação do grau de maturidade em GP & Sim & Sim \\
\hline Avaliação da integração dos processos & Sim & Não \\
\hline Questionários estruturados & Sim & Sim \\
\hline Facilidade de aplicação & Sim & Sim \\
\hline Avaliação da excelência & Sim & Não \\
\hline Identificação das competências dos indivíduos & Sim & Sim \\
\hline Ênfase na aderência estratégica com a organização & Não & Sim \\
\hline Aplicação & $\mathbf{K P M M M}^{\circledR}$ & $\mathrm{OPM}^{\circledR}{ }^{\circledR}$ \\
\hline Avaliação do gerenciamento de programas & Não & Sim \\
\hline Avaliação do gerenciamento de portfólios & Não & Sim \\
\hline Suporta a melhoria contínua & Sim & Sim \\
\hline Limitações & KPMMM $^{\circledR}$ & $\mathrm{OPM}^{\circledR}$ \\
\hline Comparações percentuais carecem de rigor estatístico & Sim & Sim \\
\hline Pouca ênfase nas equipes de projetos & Sim & Sim \\
\hline Avaliação da maturidade em escala discreta & Sim & Não \\
\hline Avaliação da maturidade em escala contínua & Não & Sim \\
\hline
\end{tabular}

Fonte: adaptado de Khoshgofar e Osman (2009) e Carvalho et al (2005) 
Ao analisar o Quadro 5 percebe-se, no que se refere à aplicação, que as duas métodos podem ser consideradas complementares, já que existem áreas que são cobertas por uma e não pela outra.

Com relação às limitações, tem-se pouca ênfase na avaliação das equipes dos projetos, pelo fato de uma abordagem realizar a avaliação de maturidade em uma escala num contínuo, uma vez que a OPM $3^{\circledR}$ avalia em porcentagem, e a $\mathrm{KPMMM}^{\circledR}$ ser discreta, conforme apresentado no Quadro 4. Há também, na literatura, questionamento quanto à validação estatística dos resultados.

Os resultados e discussões apresentados, referentes às semelhanças e diferenças dos métodos $\mathrm{OPM} 3^{\circledR} \mathrm{e} \mathrm{KPMMM}^{\circledR}$ para avaliação de maturidade em gerenciamento de projetos, podem ser interpretados como um indicativo da abrangência dessa avaliação nas organizações.

\section{Conclusão}

O presente estudo buscou realizar análise comparativa dos métodos OPM3 ${ }^{\circledR} \mathrm{e}$ $\mathrm{KPMMM}^{\circledR}$, sua aplicação e eventuais limitações para aplicação com base em pesquisa bibliográfica, os resultados foram apresentados e discutidos na subseção 4.1, com apoio dos Quadros 4 e 5. Apresenta-se uma síntese das duas métodos no Quadro 6.

A análise das informações e dados coletados, tabulados e discutidos na subseção 4.1 e sumarizados nos Quadros 4 e 5 corroboram duas conclusões obtidas por Carvalho et al (2005): a) as métodos $\mathrm{KPMMM}^{\circledR}$ e OPM3 ${ }^{\circledR}$ apresentam complementaridades, que podem ser exploradas para aplicação nas organizações; e b) as duas abordagens apresentam técnicas e parâmetros para avaliar a institucionalização do gerenciamento de projetos, mas não contemplam de maneira explícita a avaliação das competências da organização e dos indivíduos.

Durante o estudo e discussão, conforme apresentado no Quadro 6, chegou-se a outras conclusões relevantes e complementares às anteriores:

a) $\mathrm{O}$ método $\mathrm{OPM} 3^{\circledR}$, além das melhores práticas, é baseada nos padrões publicados e revisados a cada quatro anos pelo $\mathrm{PMI}^{\circledR}, P M B O K$ Guide ${ }^{\circledR}$, o padrão para Gerenciamento de Programas ${ }^{\circledR}$ e o padrão para Gerenciamento de Portfólios ${ }^{\circledR}$, $\left(\mathrm{PMI}^{\circledR}, 2008\right)$;

b) Para uma primeira avaliação da maturidade em gerenciamento de projetos, a método $\mathrm{KPMMM}^{\circledR}$ parece ser mais acessível e flexível para aplicação quando 
comparada com a OPM $3^{\circledR}$. Por outro lado, quando se faz necessário avaliar a gestão de programas ou portfólios, a método $\mathrm{KPMMM}^{\circledR}$ não suporta, e é necessário utilizar a método OPM $3^{\circledR}$.

Quadro 6 - Síntese comparativa dos métodos $\mathrm{KPMMM}^{\circledR}$ e OPM3 ${ }^{\circledR}$

\begin{tabular}{|c|c|c|}
\hline Características & $\mathbf{K P M M M}^{\circledR}$ & $\mathrm{OPM}^{\circledR}$ \\
\hline Origem & Desenvolvido por Kerzner & Desenvolvido PMI $^{(\circledR)}$ \\
\hline Fundamento & Alinhado PMBOK ${ }^{\circledR}$ Guide & $\begin{array}{l}\text { Melhores práticas e padrões } \\
\text { do PMI }{ }^{\circledR}\end{array}$ \\
\hline Níveis & Abrange cinco níveis & Abrange quatro níveis \\
\hline Método de avaliação & Questionários & Questionários \\
\hline Aplicação & Qualquer organização & Qualquer organização \\
\hline Escala de avaliação & Discreta & Contínua \\
\hline Avalia a Gestão de Projeto & Sim & Sim \\
\hline Avalia competências & Individuais dos GP & Individuais dos GP \\
\hline $\begin{array}{l}\text { Avaliação Processo Gestão dos } \\
\text { Programas }\end{array}$ & Não & Sim \\
\hline $\begin{array}{l}\text { Avaliação Processo Gestão do } \\
\text { Portfólio }\end{array}$ & Não & Sim \\
\hline Realização da avaliação & Papel ou on line & Papel ou on line \\
\hline Avalia as equipes de Projeto & Não & Não \\
\hline Foco principal & $\begin{array}{l}\text { Institucionalização do } \\
\text { gerenciamento de projetos }\end{array}$ & $\begin{array}{l}\text { Institucionalização do } \\
\text { gerenciamento de projetos }\end{array}$ \\
\hline Suporta melhoria contínua & Sim & Sim \\
\hline $\begin{array}{l}\text { Avalia o alinhamento estratégico dos } \\
\text { projetos }\end{array}$ & $\begin{array}{l}\text { Não de forma direta e } \\
\text { estruturada }\end{array}$ & $\begin{array}{l}\text { Sim, ao avaliar o alinhamento } \\
\text { de programas e portfólios. }\end{array}$ \\
\hline
\end{tabular}

Fonte: Autoria própria (2013)

Com a realização deste estudo, percebeu-se a sugestão de em estudo futuro, analisar a viabilidade de se desenvolver uma abordagem unificada para avaliação da maturidade das organizações no gerenciamento de projetos, com base nos métodos existentes, independe da abordagem de gerenciamento de projetos adotada pelas organizações.

\begin{abstract}
Project management has been an approach with accelerated expansion in the last three decades in organizations of various market segments in Brazil and worldwide. Despite the broad dissemination and use, it is still high failure rate or partial success in implementing developments. As this field of study is recent, led to question whether this index is due to the immaturity of the organizations in the use of knowledge, techniques and skills in this area. This study, conducted from literature, aims to compare and identify the conceptual differences between the models Organizational Project Management Maturity Model ${ }^{\circledR}\left(\mathrm{OPM}^{\circledR}\right)$ and Kerzner Project Management Maturity Model ${ }^{\circledR}\left(\mathrm{KPMMM}^{\circledR}\right)$ used to assess the management maturity projects in organizations. Comparison of OPM $3^{\circledR}$ and $\mathrm{KPMMM}^{\circledR}$ and your methods based on literature review, shows that there is complementarity between the two approaches and some differences, which enables the $\mathrm{KPMMM}^{\circledR}$ model can be applied in combination with the OPM $3^{\circledR}$, thereby maximize results while defining a focus for the first analysis, which allows save, resources, and reduce the time to get indications for improvement.
\end{abstract}


Key words: project manager maturity; methodology OPM $3{ }^{\circledR}$; methodology $\mathrm{KPMMM}^{\circledR}$.

\section{Referências}

AURÉLIO. Novo dicionário eletrônico Aurélio. Versão 5. 11ª . 3 ed. São Paulo: Positivo Informática, 2004.

ANDERSEN, E. S.; JESSEN, S. A. Project maturity in organizations. In: International Journal of Project Management. Sandvika: v. 21, p. 457-461, 2003.

ARCHIBALD, R. D. State of the art of project management - 2005-2010. Keynote presentation to the PMI ${ }^{\circledR}$ Milwakee/Southeast Wisconsin Chapter, Professional Development Day, Out./2005. Disponível em:

$<$ http://russarchibald.com/recent-papers-presentations/state-of-the-art/state-art-pm-in-2010-milwaukee/>. Acesso em: 25/maio/2012.

CARVALHO, M. M. et al. Equivalência e completeza: análise de dois modelos de maturidade em gestão de projetos R. Adm. São Paulo, v. 40, n.3, p.289-300, jul.-ago.-set./2005.

CARVALHO, M. M.; RABECHINI, R. (org.) Gerenciamento de projetos na prática: casos brasileiros. v. 1.1. ed. 3 reimp. São Paulo: Atlas, 2010.

Construindo competências para gerenciar projetos: teoria e casos. 2. ed. São Paulo: Atlas, 2008.

CIRILO, F. Proposta de procedimento para ampliar a maturidade em gerenciamento de projetos através das melhores práticas. 2010. 104 f. Dissertação (Mestrado em Engenharia Mecânica). Faculdade de Engenharia Mecânica - UNICAMP, Campinas.

DINSMORE, P. C.; CAVALIERI, A. (org.). Como se tornar um profissional em gerenciamento de projetos: livro-base de preparação para certificação Project Management Professional (PMP). 3. ed. Rio de Janeiro: Qualitymark, 2009.

HERKENHOFF, D. A.; F., FIGUEIREDO, A. D. F.; LIMA, G. B. Proposta unificadora de níveis de maturidade em gerenciamento de projetos. In: VII Congresso Nacional de Excelência em Gestão. 12 e 13 de Agosto, 2011.

HOUASSIS. Dicionário eletrônico. Instituto Antonio Houassis. Rio de Janeiro: Objetiva, 2009.

IBBS, C. W.; KWAK, Y. H. Assessing project management maturity. In: Project Management Journal. v.31, $\mathrm{n}^{\mathrm{o}} .1, \mathrm{p} .32-43, \mathrm{mar} / 2000$.

KERZNER, H. Gestão de projetos: as melhores práticas. Porto Alegre: Artmed, 2002.

Using the project management maturity model: strategic planning for project management. 2 . ed. New Jersey: John Wiley \& Sons, Inc, 2005.

Project management: a system approach to planning; schedulling and controlling. 10. ed. New Jersey: John Wiley \& Sons, Inc, 2009.

KHOSHGOFTAR, M.; OSMAN, O. Comparison of Maturity Models. New Jersey: John Wiley \& Sons, Inc, 2009. crossref

LUKOSEVICIUS, A. P.; CAMPOS FILHO, L. A. N. Maturidade organizacional e desempenho de projetos no setor naval brasileiro. In: Revista Produção On Line, Florianópolis: v. 8, nº 1, mar./2008. cross ref

MAXIMIANO, A. C. A. Administração de projetos: como transformar ideias em resultados. 4 ed. São Paulo: Atlas, 2010

MARCONI, M. A.; LAKATOS, E. M. Fundamento de método científica. 6. ed. São Paulo: Atlas, 2005.

$\mathrm{PMI}^{\circledR}$ - PROJECT MANAGER INSTITUTE.. A guide to the project manager body of knowledge. $\left(\mathrm{PMBOK}^{\circledR}\right.$ Guide) - Fourth Edition 4. ed. Pennsylvania: Project Manager Institute, Inc., 2008a 
PRADO, D. S. Organizational project management maturity model: OPM $3{ }^{\circledR}$ Guide. 2. ed. Pennsylvania (USA): Project Manager Institute, Inc., 2008.

Maturidade em gerenciamento de projetos. In: Série Gerência de Projetos, v. 7, 2. ed. Novo Lima: INDG Tecnologia e Serviço Ltda, 2010.

RABECHINI, Roque Jr.; PESSÔA, Marcelo Schneck de Paula. Um modelo estruturado de competências e maturidade em gerenciamento de projetos. Revista Produção, v. 15, n. 1, p. 034-043, Jan./Abr. 2005

RABECHINI, R. A estruturação de competências e maturidade em gerenciamento de projetos. (2012) [s.n.t]. Disponível em: 〈http://www.fundacaofia.com.br/pgtusp/eventos/seminarios/texto_roque.pdf〉. Acesso em: 25/maio/2012.

SEGISMUNDO, André ; CARVALHO, Marly Monteiro de. Maturidade em gestão de projetos : análise comparativa em 3 unidades de negócio do setor automobilístico. IN : XVI ENGEP - Encontro Nacional de Engenharia de Produção. Fortaleza : 9 a 11 de outubro de 2006. Díspon’viel em : < http://www.labceo.com.br/bibliografia/archive/files/g-6 7cc1ee6dec.pdf> . Acesso em 25 maio 2012.

SCHLICHTER, J. PMI's organizational project management maturity model: emerging standards proceedings of the Project Management Institute. In: ANNUAL SEMINARS \& SYMPOSIUM, Nov. 1-10, 2001, Nashville, Tenn., USA. Upper

SILVA, E. L.; MENEZES, E. M. Método da pesquisa e elaboração de dissertação. 4 e. Rev. Atual. Florianópolis: UFSC, 2005.

VALLE, A. B.; et al. Série gerenciamento de projetos: fundamentos do gerenciamento de projetos. 2 ed. Rio de Janeiro: FGV, 2010.

VERGOPIA, C. Project review maturity and project performance: an empirical case study. $2008.242 \mathrm{f}$. Tese (Doctor of Philosophy in the Department of Industrial Engineering and Management Systems in the College of Engineering and Computer Science). University of Central Florida, Orlando, Florida. Disponível em: <http://etd.fcla.edu/CF/CFE0002401/Vergopia_Catherine_A_200812_PhD.pdf>. Acesso em: 24/julho/2012.

WEBSTER J. F. M.; KNUTSON, J. What is project management? project management concepts and methodologies. In: DINSMORE, P. C.; CABANIX-BREWIN, J. The AMA Handbook of Project Management, 2. ed. New York: American Management Association (AMA), 2006.

\section{Dados dos autores:}

Nome completo: Evandro Luiz de Oliveira

Filiação institucional: Universidade de Taubaté (UNITAU)

Departamento: PPGA - Programa de Pós Graduação em Administração

Função ou cargo ocupado: Professor

Endereço completo para correspondência: Vila Nossa Senhora das Graças, 65 - Vila da Graça

- Taubaté - SP CEP: 12060-590

Telefones para contato: $(+5512)$ 999781-1138

E-mail:evandro.l.o@ig.com.br 
Nome completo: Edson Aparecida de Araujo Querido Oliveira

Filiação institucional: Universidade de Taubaté (UNITAU)

Departamento: ECA - Departamento de Economia, Contabilidade e Administração

Função ou cargo ocupado: Coordenação Geral do Programa de Pós Graduação em Administrarão - PPGA da UNITAU

Endereço completo para correspondência: Rua Expedicionário Ernesto Pereira, Portão 2Taubaté - SP CEP: 12020-330

Telefones para contato: $(+5512)$ 999781-1138

E-mail:edson@unitau.br

Submetido em: 02-05-2015

Aceito em: 14-09-2015 